\title{
Ureteral fibroepithelial polyps: A modern approach to management
}

Dylan Hoare, MD; Michael Hobart, MD, FRCSC

Division of Urology, Misericordia Community Hospital, University of Alberta, Edmonton, AB, Canada

Cite as: Can Urol Assoc J 2018 March 19; Epub ahead of print. http://dx.doi.org/10.5489/cuaj.5001

Published online March 19, 2018

In November 2016, a 45-year-old male presented with undifferentiated left flank pain to his family physician. There was no evidence of urosepsis or renal dysfunction. He had a remote history of isolated gross hematuria and a negative ultrasound from 2006, but otherwise no appreciable risk factors for urinary pathology. Initial assessment with ultrasound was once again inconclusive. The abdomen was further evaluated with a triphasic renal CT scan, confirming the presence of a 7 x $25 \mathrm{~mm}$ central filling defect within the proximal right ureter (Figures 1-2). Based on the tubular, elongated nature of the filling defect, the presumptive diagnosis of a ureteral fibroepithelial polyp (UFP) was made. Both semi-rigid and flexible ureteroscopy were performed. A single stalk was identified at the level of the UPJ from which the polyp extended (Figure 3). Holmium laser was used to incise and photocoagulate the stalk, after which it was excised using rigid biopsy forceps and delivered through the semi-rigid ureteroscope (Figure 4). After excision, the base was fulgurated with a laser frequency of $40 \mathrm{~Hz}$. The specimen was delivered for permanent section and histopathologic analysis. Pathology was read consistent with a benign fibroepithelial polyp, demonstrating a loose, predominantly fibrovascular stroma covered by a benign urothelium. With increasing comfort of endourologic modalities, the ureteroscopic management of UFPs at the UPJ represents a mainstay of therapy in modern day urology. In addition, it circumvents the unnecessary morbidity of open and laparoscopic procedures. 
Figures and Tables

Fig.1. Triphasic computerized tomography scan of a coronal image of central ureteric filling defect.

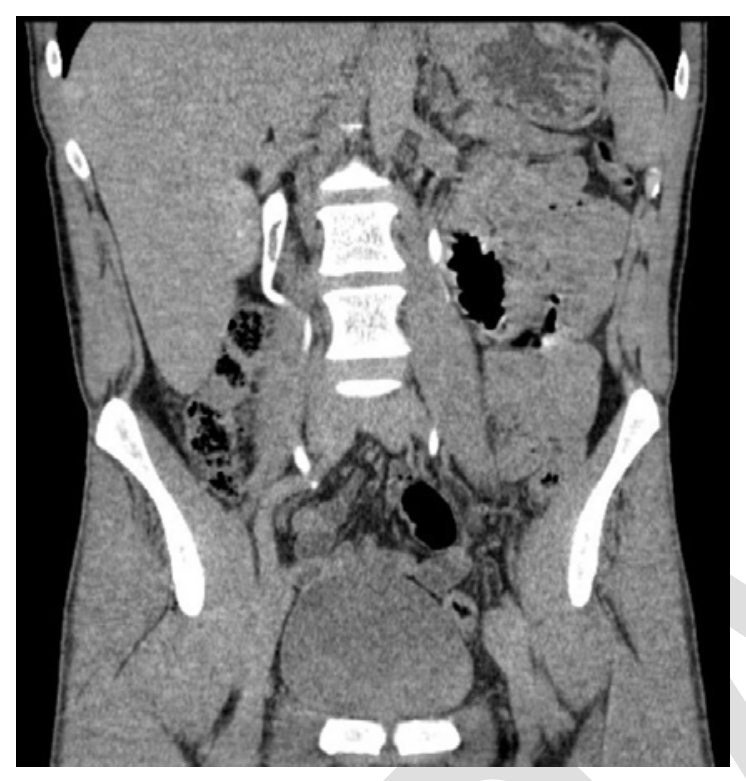

Fig. 2. Triphasic computerized tomography scan of an axial image of central ureteric filling defect.

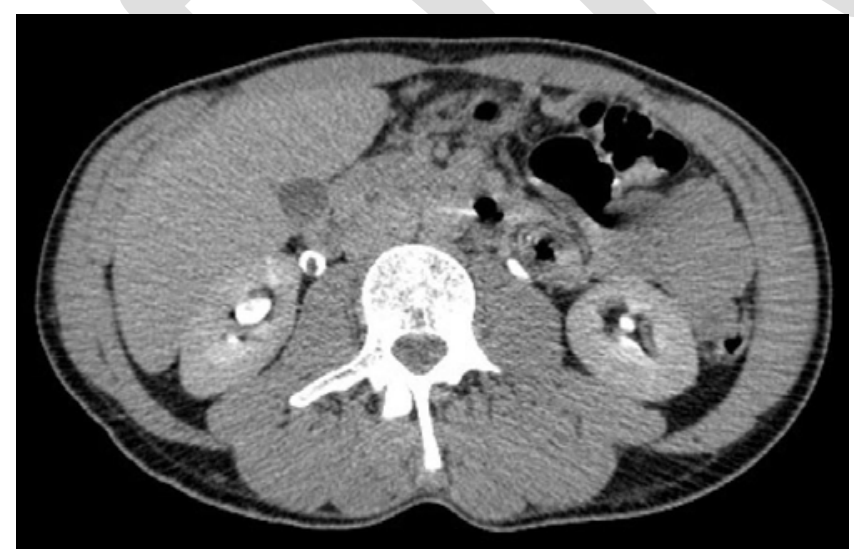


Fig. 3. Endoscopic visualization of ureteric lesion.

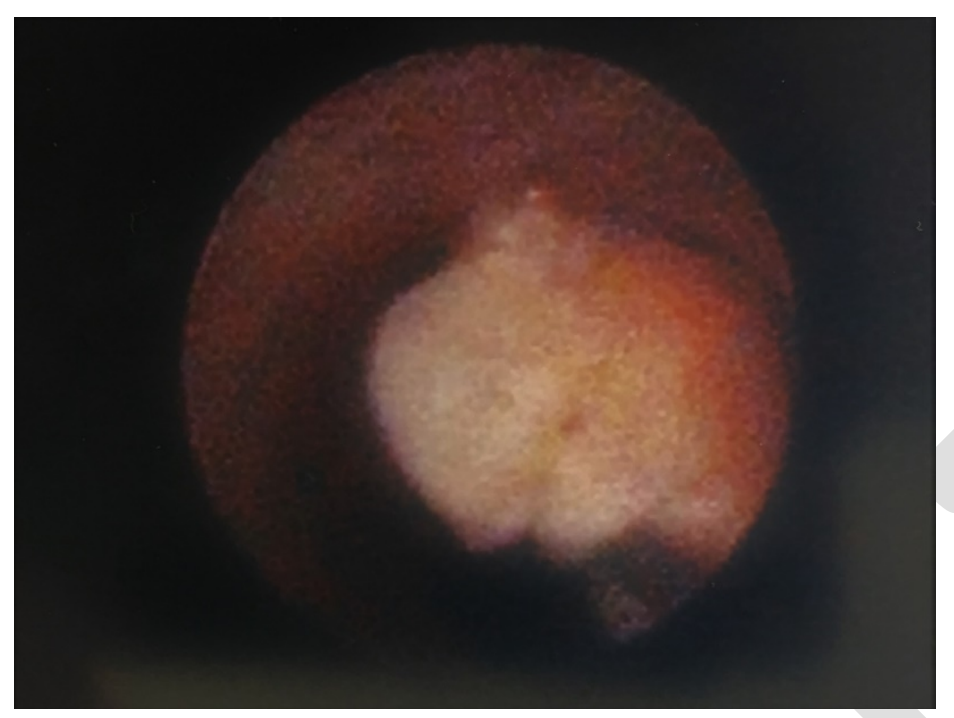

Fig. 4. Gross pathology of ureteric lesion.

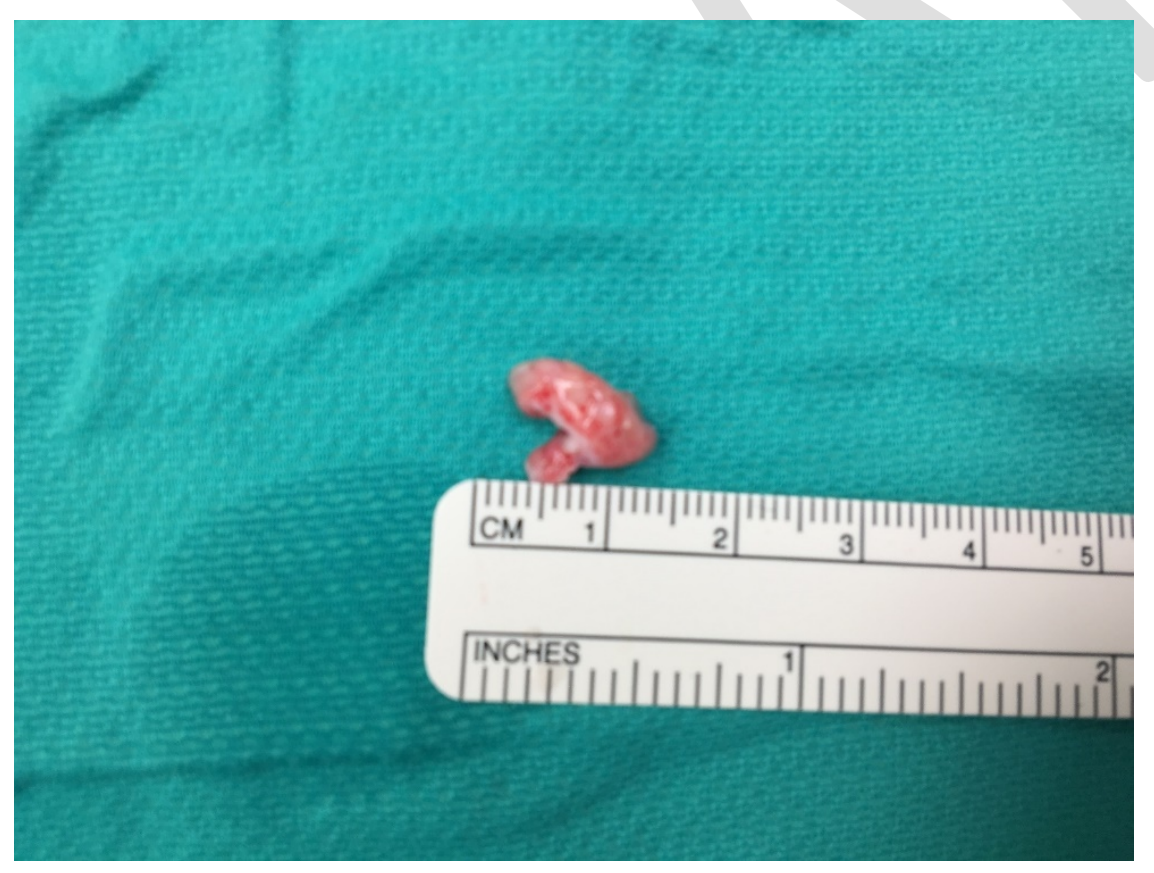

